\title{
AN APPLICATION OF GRAPH THEORY TO ALGEBRA
}

\author{
RICHARD G. SWAN ${ }^{1}$
}

1. Introduction. If $A_{1}, \cdots, A_{k}$ are any $n \times n$ matrices we define

$$
\left[A_{1}, \cdots, A_{k}\right]=\sum \operatorname{sgn}(\sigma) A_{\sigma 1} \cdots A_{\sigma k}
$$

the sum being taken over all permutations $\sigma$ of the integers 1,2 , $\cdots, k$. The following remarkable theorem was proved by Amitsur and Levitzki $[1] .^{2}$

Theorem 1. If $A_{1}, \cdots, A_{2 n}$ are any $n \times n$ matrices with entries in any commutative ring, then $\left[A_{1}, \cdots, A_{2 n}\right]=0$.

It is easy to see that the number $2 n$ is the best possible. In other words if $k<2 n$, we can find $k$ matrices $A_{1}, \cdots, A_{k}$ all $n \times n$ such that $\left[A_{1}, \cdots, A_{k}\right] \neq 0$.

The original proof of the theorem [1] was elementary but very complicated. In attempting to simplify this proof, I found a more transparent proof based on the use of graph theory. ${ }^{3}$ One advantage of this approach is that complicated algebraic definitions can be replaced by much simpler geometric definitions merely by drawing a picture of the appropriate graph. Before stating the graph theoretic theorem which implies Theorem 1, I will give some elementary definitions and lemmas from graph theory.

\section{Graph theory.}

Definition 1. An oriented graph $\Gamma$ consists of a set of points (called vertices) and a set of oriented segments (called edges) joining certain of these vertices. Examples of parts of graphs are shown in Figures 1 to 10 below.

Note that we allow a vertex to be joined to itself and we also allow two vertices to be joined in many ways. Let $V$ be the number of vertices of the graph and $E$ the number of edges. I will only be concerned here with the case in which $E$ and $V$ are finite.

If $P$ is any vertex of $\Gamma$, the order of $P$ is defined to be the total number of edges beginning or ending at $P$. An edge which joins $P$ to itself is to be counted twice. The flux of $P$ is defined to be the num-

Received by the editors April 2, 1962.

1 The author is an Alfred P. Sloan fellow.

2 For an interesting discussion of this result, see [4].

${ }^{3}$ The connection between Theorem 1 and graph theory was also pointed out by M. Schutzenberger [3, p. 167]. A proof using his idea was recently worked out by C. Y. Chao and Schutzenberger. Their proof seems to be quite different from mine. 
ber of edges beginning at $P$ minus the number of edges ending at $P$.

From now on, I will assume that $\Gamma$ has no vertices of order 0 .

Definition 2. If $P$ and $Q$ are vertices of $\Gamma$, a unicursal path $\omega$ from $P$ to $Q$ consists of an enumeration $e_{1}, e_{2}, \cdots, e_{E}$ of all the edges of $\Gamma$ such that:

(a) $e_{1}$ begins at $P$.

(b) $e_{E}$ ends at $Q$.

(c) For $1 \leqq i<E$, the initial point of $e_{i+1}$ is the terminal point of $e_{i}$. Intuitively, a unicursal path is a method of walking from $P$ to $Q$ along the edges so that every edge is traversed just once, and in the proper direction.

Euler [2] gave some necessary conditions for the existence of unicursal paths on unoriented graphs. These have immediate analogues in the oriented case.

Proposition 1. If there is a unicursal path from $P$ to $Q$, then:

(a) $\Gamma$ is connected.

(b) Every vertex other than $P$ and $Q$ has flux 0 .

(c) If $P=Q$, then $P$ has flux 0 .

(d) If $P \neq Q$, then $P$ has flux +1 and $Q$ has flux -1 .

Proof. The necessity of (a) is clear. That of (b) follows immediately from the fact that we must leave each vertex the same number of times that we enter it. The same argument applies to (c), while (d) follows from the fact that in this case, we leave $P$ one more time than we enter it, the reverse holding for $Q$.

Now, let us choose a definite (arbitrary) ordering for the edges of $\Gamma$, say by numbering them. Each unicursal path $\omega=\left(e_{1}, \cdots, e_{E}\right)$ then gives a permutation of the edges of $\Gamma$. Define $\epsilon(\omega)$ to be the sign of this permutation. We can now state the main theorem.

Theorem 2. Suppose $E \geqq 2 V$. Let $P$ and $Q$ be any fixed vertices of $\Gamma$ (not necessarily distinct). Then the number of unicursal paths $\omega$ from $P$ to $Q$ with $\epsilon(\omega)=+1$ is equal to the number of unicursal paths $\omega$ from $P$ to $Q$ with $\epsilon(\omega)=-1$.

Before proving this theorem, I will show how it implies Theorem 1.

3. Theorem 2 implies Theorem 1 . For $1 \leqq i, j \leqq n$, let $e_{i, j}$ denote the $n \times n$ matrix unit with $(i, j)$ th entry 1 , the remaining entries being 0 . These $e_{i, j}$ multiply according to the rule:

$$
e_{i, j} e_{k, l}= \begin{cases}0 & \text { if } j \neq k \\ e_{i, l} & \text { if } j=k .\end{cases}
$$

Clearly, any $n \times n$ matrix is a linear combination of such matrix 
units. Since $\left[A_{1}, \cdots, A_{2 n}\right]$ is multilinear, it is sufficient to prove Theorem 1 for the case where all the $A_{i}$ are matrix units.

Suppose then that $A_{1}, \cdots, A_{2 n}$ are all matrix units. Define an oriented graph $\Gamma$ as follows: $\Gamma$ has $n$ vertices $P_{1}, \cdots, P_{n}$. $\Gamma$ has one edge $e_{i}$ for each $A_{i}$ where, if $A_{i}=e_{j, k}, e_{i}$ has initial point $P_{j}$ and terminal point $P_{k}$. Clearly $E=2 V$ for this graph.

The multiplication rule (1) now shows that a product $A_{\sigma 1} \cdots A_{\sigma 2 n}$ has a nonzero $(i, j)$ th entry if and only if the corresponding sequence of edges $e_{\sigma 1}, \cdots, e_{\sigma 2 n}$ is a unicursal path from $P_{i}$ to $P_{j}$. In this case, the $(i, j)$ th entry is 1 . Since we are adding up all these products $A_{\sigma 1} \cdots A_{\sigma 2 n}$ each prefixed with the sign of $\sigma$, Theorem 2 shows immediately that the $(i, j)$ th entry of $\left[A_{1}, \cdots, A_{2 n}\right]$ for 0 for any $i$ and $j$.

4. Preliminaries to the proof. Before giving the main argument, I will make some preliminary remarks.

(1) Suppose that the two edges $e$ and $e^{\prime}$ of $\Gamma$ have the same initial points and the same terminal points. Then the theorem holds for $\Gamma$. To see this, we merely observe that, given any unicursal path $\omega$, we can form a new one $\omega^{\prime}$ by interchanging $e$ and $e^{\prime}$. Since we have performed a transposition, $\epsilon(\omega)=-\epsilon\left(\omega^{\prime}\right)$.

(2) The theorem is true if $\Gamma$ is not connected since then there are no unicursal paths at all.

(3) If the theorem is true for the case $E=2 \mathrm{~V}$, it is also true for the case $E>2 \mathrm{~V}$. To see this, we modify $\Gamma$ by introducing $k=E-2 \mathrm{~V}$ new vertices and edges as in Figure 1, getting a new graph $\Gamma^{\prime}$.

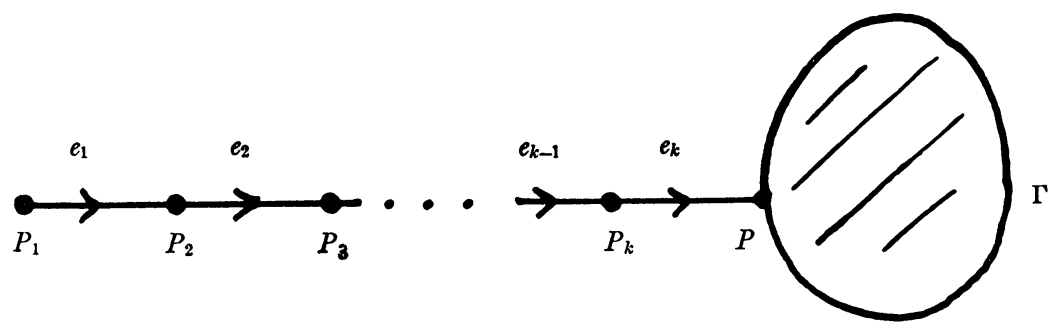

FIGURE 1

This new graph satisfies the condition $E=2 \mathrm{~V}$. Clearly there is a 1-1 correspondence between unicursal paths from $P_{1}$ to $Q$ on $\Gamma^{\prime}$ and unicursal paths from $P$ to $Q$ on $\Gamma$. This correspondence preserves the signs $\epsilon(\omega)$.

(4) If the theorem is true for the case where $E=2 \mathrm{~V}$ and all vertices have flux 0 , it is then true in general. To see this, we note that 
if not all vertices have flux 0 , the only nontrivial case is that where all have flux 0 but $P$ which has flux +1 and $Q$, which has flux -1 . Proposition 1 shows that there are no unicursal paths from $P$ to $Q$ in any other case. We now define a new graph $\Gamma^{\prime}$ by adding two edges and a vertex as in Figure 2.

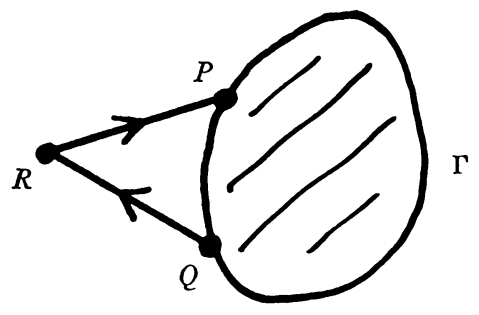

FIGURE 2

There is clearly a 1-1 correspondence between unicursal paths from $P$ to $Q$ on $\Gamma$ and unicursal paths from $R$ to $R$ on $\Gamma^{\prime}$. Again $\epsilon(\omega)$ is obviously preserved by this correspondence.

5. Proof of Theorem 2. By the remarks of $\S 4$, we can assume $E=2 V$ and that all vertices have flux 0 . All the transformations of $\Gamma$ performed below will preserve these conditions. We now proceed by induction on $V$. For $V=1$, the theorem is trivial, e.g. by Remark (1) of $\S 4$. Let us assume that $V>1$. There are three cases to consider.

Case 1. $\Gamma$ contains the configuration of Figure 3.

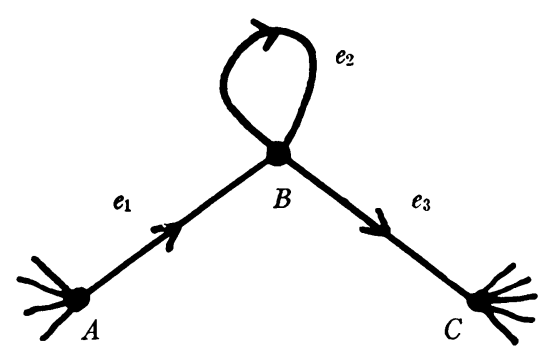

FigURE 3

If $P=B$, note that every unicursal path must begin or end with $e_{2}$. By moving $e_{2}$ from the beginning to the end or vice versa, we get a correspondence $\omega \leftrightarrow \omega^{\prime}$ between unicursal paths. Since $\epsilon(\omega)=-\epsilon\left(\omega^{\prime}\right)$, the theorem holds in this case.

If $P \neq B$, we replace the configuration made up of $e_{1}, e_{2}, e_{3}$, and $B$ by a single edge $e$ as in Figure 4, getting a new graph $\Gamma^{\prime}$. There is 


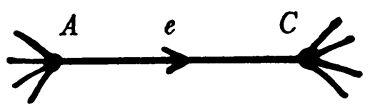

Figure 4

obviously a 1-1 correspondence between unicursal paths from $P$ on $\Gamma$ and on $\Gamma^{\prime}$. It is trivial to check that this correspondence preserves $\epsilon(\omega)$. Now $\Gamma^{\prime}$ has fewer vertices than $\Gamma$ so the induction hypothesis applies. This is the only case in which the induction hypothesis will be used. The other cases will be treated by reducing them to Case 1 .

Case $2 . \Gamma$ has a vertex of order 2 .

Since $E>V$, not all vertices have order 2. By Remark (2) of $\$ 4$, we may assume $\Gamma$ connected. Therefore, $\Gamma$ contains a configuration as in Figure 5 where $A$ has order greater than 2.

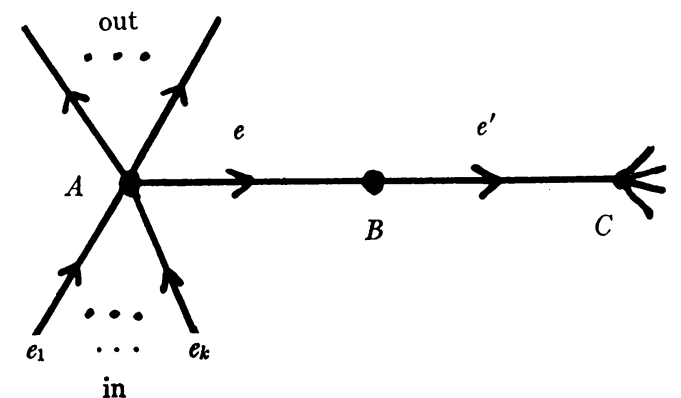

Figure 5

For each edge $e_{i}, i=1, \cdots, k$ terminating at $A$, define a new graph $\Gamma_{i}$ by making the transformations indicated in Figure 6. The part of $\Gamma$ not shown should, of course, remain unaltered.
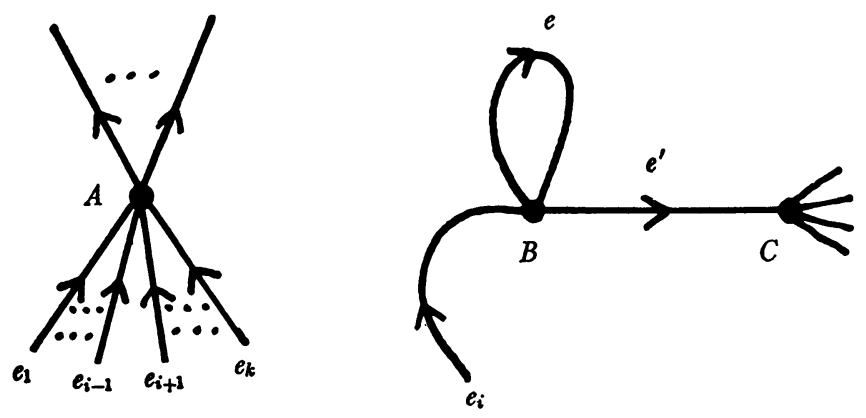

Figure 6 
In any unicursal path on $\Gamma$, one of the $e_{i}$ must immediately precede $e$. Consequently, this path is also unicursal on $\Gamma_{i}$ and on no other $\Gamma_{j}$, $j \neq i$. Conversely, any unicursal path on any $\Gamma_{i}$ is also unicursal on $\Gamma$. Because of this correspondence, it is sufficient to prove the theorem for each $\Gamma_{i}$. But, each $\Gamma_{i}$ satisfies the condition of Case 1 .

Case 3. Cases 1 and 2 do not apply.

Since each vertex has flux 0 , it must have even order. Consequently, it has order $\geqq 4$ since Case 2 does not apply. Now, the average order of the vertices is $2(E / V)=4$, since each edge has two endpoints. This implies that each vertex has order exactly 4 . Therefore, $\Gamma$ contains the configuration of Figure 7.

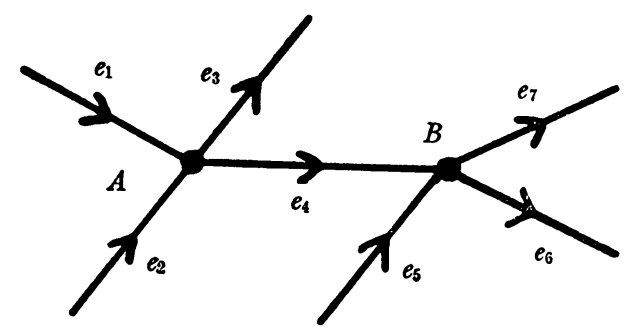

FIGURE 7

We now try the construction used in Case 2. This gives us the graphs $\Gamma_{i}, i=1,2$ of Figure 8 . Here we let $i^{\prime}=2$ if $i=1$ and $i^{\prime}=1$ if $i=2$.

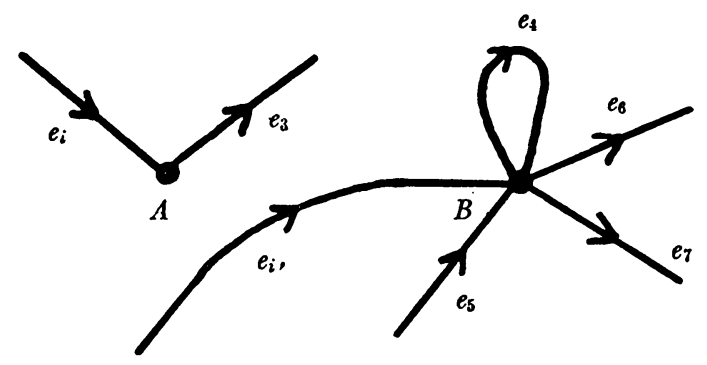

FIGURE 8

As in Case 2, every unicursal path on $\Gamma$ is unicursal on exactly one of $\Gamma_{1}$ and $\Gamma_{2}$. However, there are spurious unicursal paths on $\Gamma_{i}$ which enter $B$ by $e_{i}$, but then leave by $e_{6}$ or $e_{7}$ without going around $e_{4}$. Such paths are not unicursal on $\Gamma$. However, they all contain a subpath $e_{5} e_{4} e_{j}$ where $j=6$ or 7 . Consequently, they are exactly those paths 
which are unicursal on one of the graphs $\Gamma_{j}^{\prime}, j=6,7$ of Figure 9. Here, as before, $j^{\prime}=7$ if $j=6$, and $j^{\prime}=6$ if $j=7$.

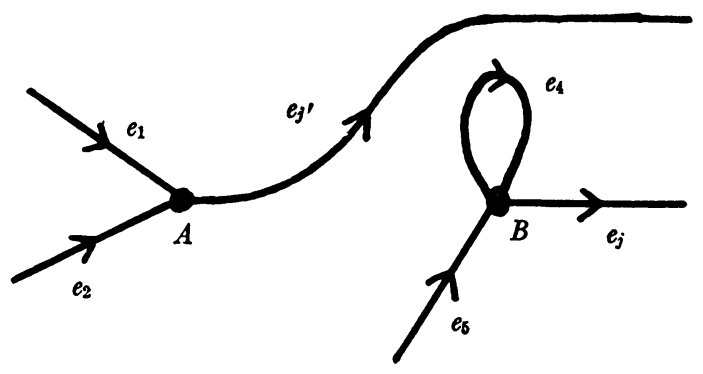

Figure 9

Now, the unicursal paths on the $\Gamma_{i}, i=1,2$ are exactly those unicursal on either $\Gamma$ or on some $\Gamma_{j}^{\prime}, j=6,7$. Since Case 2 applies to the $\Gamma_{i}$ and Case 1 applies to the $\Gamma_{j}^{\prime}$, it follows that the theorem holds for $\Gamma$.

6. An example. To show that the condition $E \geqq 2 V$ is really needed in Theorem 2, consider the following graph $\Gamma$. Here $E=2 V-1$. There

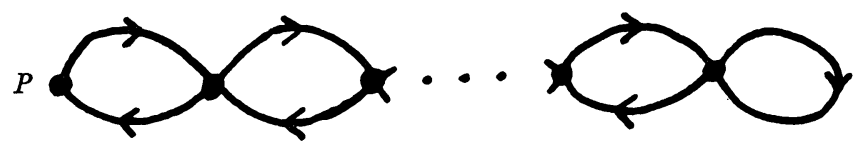

FIgURE 10

is clearly only one unicursal path from $P$ to $P$.

\section{REFERENCES}

1. S. A. Amitsur and J. Levitzki, Minimal identities for algebras, Proc. Amer. Math. Soc. 1 (1950), 449-463.

2. W. W. Rouse Ball, Mathematical recreations and essays, Chapter IX, Macmillan, New York, 1947.

3. C. Berge, Theorie des graphes et ses applications, Dunod, Paris, 1958.

4. B. Kostant, $A$ theorem of Frobenius, a theorem of Amitsur-Levitzki, and cohomology theory, J. Math. Mech. 7 (1958), 237-264.

University of Chicago 